\title{
ORGANIZACIÓN INDUSTRIAL A TRAVÉS DE REDES DE SUBCONTRATACIÓN. UNA ALTERNATIVA A LAS ACTIVIDADES MEXICANAS DE MAQUILA ${ }^{1}$
}

\author{
Por \\ Alejandro Mungaray Lagarda*
}

\begin{abstract}
RESUMEN
En este artículo se discuten aspectos básicos dela organización institucional de la economía, partiendo de una visión sistémica de la empresa. Se expone el moderno sistema de organización industrial flexible y se relaciona con la implementación de redes de pequeñas empresas que manejan escalas óptimas reducidas, resaltando, además, la importancia del aprendizaje industrial.

De lo anterior, se desprende el planteamiento de la formación de redes de subcontratación como una alternativa de organización para la industria maquiladora mexicana, en especial, la ubicada en el norte del país.
\end{abstract}

\begin{abstract}
In this article, basic aspects of the institutional organization of the economy are discussed, departing from a sistematic view of the company. The modern system of the flexible industrial organization is exposed and linked to the implementation of small businesses that manage reduced optimum scales, rebounding, also, the importance of the industrial learning. From all this, it is detached the proposal of the formation of nets of subcontractions as an alternative of organization for the Mexican industry of the maquiladora, in particular, that located in the North of the country.
\end{abstract}

\section{INTRODUCCIÓN ${ }^{2}$}

La intensa discusión que durante años se ha desarrollado en torno a la industria maquiladora de exportación, ubicada principalmente en la frontera

1 Ponencia presentada en el Seminario Nacional sobre Alternativas de la Economía Mexicana, organizado por el Instituto de Investigaciones Económicas de la UNAM, el 18 de noviembre de 1993, México, D.F.

* Académico de la Facultad de Economía, Universidad Autónoma de Baja California, Tijuana, B.C.

Agradezco los valiosos aportes de los profesores Clemente Ruiz Durán, Bernardo González-Aréchiga, Jorge Mattar y Julio López, así como la asistencia de Dora Elia Felician. 
norte, ha dado lugar a enfoques diversos sobre cómo es y cómo debiera ocurrir el desarrollo de este tipo de actividades. Aspecto central de esta discusión, ha sido su integración a la economía mexicana y su contribución a la formación de una base empresarial nacional.

El hecho de que durante muchos años se haya concentrado el análisis de la integración, en la proporción de insumos nacionales respecto al total de insumos utilizados por la industria maquiladora, no ha permitido que los objetivos macroeconómicos pudieran ser articulados en una política industrial integral. Hoy es evidente que el incremento del empleo y el fortalecimiento de la balanza de pagos son objetivos en los cuales se ha avanzado mucho. También es claro que la mayor integración interindustrial, el mejoramiento de la competitividad de la industria nacional, el desarrollo de la capacitación del trabajo y la transferencia de tecnología, son objetivos para los cuales no existen aún mecanismos plenamente institucionalizados que permitan aprovechar, aún más y de manera integral, el potencial tecnológico y de formación empresarial de la industria maquiladora mexicana.

Existe evidencia acerca de las oportunidades de negocios que, a partir de las actividades de maquila, han dado lugar a sectores empresariales de carácter nacional. La construcción de infraestructura (Mungaray y Álvarez, 1987), la oferta de servicios profesionales y de maquila (GonzálezAréchiga et al.,1991) son áreas perfectamente indentificadas como nichos de mercado para empresarios nacionales. En ramas industriales como la téxtil, por ejemplo, existe un mercado siempre abierto para nuevos productores.

En algunos casos en que el aprendizaje industrial ha sido rápido y eficiente, se han visto transformaciones de pequeños empresarios en grandes, ya sea en actividades industriales específicas, o como organizadores de servicios de maquila para nuevas empresas extranjeras que desean incursionar, con el menor riesgo posible, en actividades de subcontratación que mejoren sus rendimientos económicos (Mungaray, 1991:605).

Es necesario mencionar que frente a esta riqueza tecnológica, resalta la falta de estrategias efectivas de integración y coordinación que permita superar las fallas de mercado que impiden el desarrollo más amplio del empresario nacional (Ruiz D., 1991:86). El hecho de que las instituciones educativas realicen actividades de capacitación que no encuentran mercado y que las empresas requieran servicios de capacitación que no disponen regionalmente, o el hecho de que las grandes y estables empresas maquiladoras tengan que importar la casi totalidad de sus insumos mientras un buen número de pequeñas empresas nacionales sufren la falta de mercados para sus productos, son ejemplos que dan idea de la ausencia de instituciones coordinadoras de los agentes económicos; pero a la vez, de 
su importancia para convertir en oportunidades de desarrollo empresarial, aprendizaje tecnológico y generación de mayor valor agregado, las fallas del mercado generadas por información imperfecta.

En lo que sigue, se discuten los aspectos básicos de la organización institucional de la economía, a partir de una visión sistémica de la empresa. La flexibilidad de la organización empresarial moderna, se asocia a la formación de redes de pequeñas empresas, con escalas óptimas reducidas y que aprenden rápidamente a través de redes de subcontratación. Esto da lugar para plantear la formación de redes de subcontratación, como una alternativa organizacional de las actividades de maquila mexicanas, con más impacto en los objetivos de formación de empresarios, aprendizaje industrial e interacción interindustrial.

\section{ORGANIZACIÓN INSTITUCIONAL DE LA ECONOMÍA}

Las instituciones son en sí mismas acuerdos minimizadores de costos de transacción (Nabli y Nugent, 1989:11), pues en presencia de costos de transacción crecientes, se incrementan también las presiones sobre las economías de escala que es posible lograr en las empresas. El enfoque institucional de la economía considera a las empresas como las organizaciones económicas que se forman para evitar al máximo los costos de transacción. Muchas de las transacciones requeridas para lograr economías de escala, son más eficientes si se realizan internamente que a través del mercado (Pratten, 1991:5). La ineficiencia del mercado y del mecanismo de precios como asignador de recursos, se origina en el creciente dinamismo de la economía y las instituciones, y en la creciente incertidumbre que genera el difícil acceso a la información. Esto influye en que algunas decisiones de las empresas no sean eficientes.

La relacion entre mercado y empresa tiene dos vertientes. La primera se origina cuando los intercambios mediados por el mercado son sustituidos por un proceso administrativo dentro de la empresa. Ello indica que su organización interna sirve para economizar en costos de transacción que origina el mercado y afectan las economías de escala que es posible obtener. La función de producción así conformada, contrariamente a lo expresado por el pensamiento neoclásico, se orienta más a la búsqueda de menores costos de transacción que a la búsqueda de diferencias competitivas a través de la tecnología (Nabli y Nugent, 1989:9). La integración vertical de la empresa sería entonces superior, no por el gran tamaño que adquiere, sino porque la integracion misma le permite aumentar la velocidad de acceso a insumos que aumentan el nivel de producto. La segunda vertiente se origina cuando los intercambios mediados por procesos de 
administración internos, son sustituidos por un proceso de mediación a través del mercado. Coase (1937:395) apunta que la decisión de acudir al mercado ocurre cuando "los costos de organizar una transacción extra dentro de la empresa, son iguales a los costos de realizar la misma transacción por medio de un intercambio en el mercado abierto, o a los costos de organizarla en otra empresa". Las fallas de mercado que se originan cuando los compradores y vendedores son pocos, elevan los costos de transacción de las empresas y las orientan a su integración vertical, buscando economías de escala en función de su tamaño. Sin embargo, las fallas de organización interna debido al gran tamaño, elevan los costos de operación por encima de los rendimientos crecientes que es posible obtener con su funcion de producción y las orientan entonces a su desintegración horizontal.

Cuando los costos de transacción e información crecen, aun por acciones de gobierno, la acción colectiva de los agentes involucrados es necesaria para su solución. La forma y carácter de las organizaciones para la acción colectiva, dependerá en alto grado de los costos de transacción (Nabli y Nugent, 1989:446); sin embargo, normalmente estará orientada a reducir sus costos de inicio y los costos medios de largo plazo.

\section{ORGANIZACIÓN FLEXIBLE Y VISIÓN SISTÉMICA DE LA EMPRESA}

Al considerar la economía como un ambiente donde la única certidumbre es la incertidumbre y la incertidumbre un nivel desconocido de riesgo (Obrinsky, 1983:75), las empresas japonesas se organizan con base en el entendimiento y la rápida respuesta a las necesidades de los consumidores, con una oferta diversificada que permite más alternativas de elección; en su habilidad para crear nuevos mercados; para desarrollar nuevos productos y dominar tecnologías emergentes (Nonaka, 1991:96-7). Por eso es que los sistemas de entrega justo a tiempo y de automatización flexible, crean posibilidades organizacionales que superan la rígida lógica de la productividad como una función a escala, incluyendo su sistema de máquinas especializadas asociado, y permiten un mejor aprovechamiento productivo de los procesos automatizados de manufactura (Storper, 1985:272).

El paradigma de organización industrial flexible descansa en la organización funcional de redes entre grandes y pequeñas empresas a través de la subcontratación (Gerlach y Lincoln, 1992:494). Esto permite cambiar de un proceso de producción a otro en función de los signos de mercado, reduciéndose la escala óptima de producción a una cuarta parte de la requerida en la producción en masa (Ruiz y Kagami, 1993:3). Para ello, el 
aprendizaje empresarial es una condición necesaria de tal proceso, y por tanto, el aspecto organizativo es el que hace la gran diferencia.

Mientras el sistema de produccion en masa establece metas personales limitadas, donde suficientemente bueno se traduce en un aceptable número de defectos, un máximo nivel de inventario y un rango estandarizado de productos, el sistema de producción flexible establece señales explícitas de perfección, costos en constante declinación, fortalece el criterio de responsabilidad aumentando la libertad en el control del trabajo, estimula el aprendizaje profesional, la capacitacion y el desarrollo de productos y fortalece la coordinación del sistema de producción con proveedores y consumidores (Womack, Jones y Roos, 1990:13-4). Como esto requiere mucha comunicación, la tecnología descansa en la informacion.

Son cinco conceptos los que a juicio de Womack, Jones y Roos caracterizan el sistema de organización industrial flexible. El concepto de "empresa como comunidad", significa que ésta asume la responsabilidad por el empleo vitalicio y remuneraciones mayores, bajo la fórmula de recompensa combinada por creatividad y antigüedad. Esto incluye facilidades y apoyo para vivienda, recreación y educación de sus trabajadores (Womack, Jones y Roos, 1990:53). En esta visión, la fuerza de trabajo se considera como un activo fijo de largo plazo que es importante desarrollar, a fin de alcanzar una actitud crecientemente innovadora y carente de temores que permitan una automatizacion flexible completa (Baranson, 1981:24; Deutschmann, 1987:470; Hayes y Jaikumar, 1988:81).

El concepto de "trabajo en equipo" permite la organización laboral en equipos, conocidos en occidente como círculos de calidad. En un principio asumieron la responsabilidad de las tareas de limpieza, reparaciones menores y control de calidad. Posteriormente, pasaron a sugerir innovaciones al proceso y a retroalimentar a los ingenieros industriales. Este sistema de organización permite que el control del trabajo, de la calidad del producto, de la calidad y oportunidad de los inventarios y de los costos de producción, esté bajo la responsabilidad del área de producción. Y aunque existe un esquema de jerarquías, este es de equipos de trabajo a grupos a secciones, y no de individuos a supervisor a gerente de planta como en el sistema de producción en masa (Ogawa, 1984:48-9; Womack, Jones y Roos, 1990:56-7).

Abatir costos de inventarios buscando economías de escala por compras en volumen, evitar su almacenamiento en grandes almacenes y aumentar su control con una estructura administrativa que permita su oportuna entrega a la línea de producción, se logra a través de las "cadenas de oferta". La relación entre empresa y proveedor para reducir costos e innovar en calidad, es una relación de mercado sobre el principio de precio, 
calidad y oportunidad de entrega. Como las empresas orientan su esfuerzo productivo en función de la demanda, la cadena de proveedores, compuesta por un gran número de pequeñas empresas como oferentes de partes para la elaboración de productos finales, "tienen que ser capaces de producir lo que se necesite cuando se necesite" (Ogawa, 1984:104). La coordinación con los proveedores se realiza a través del flujo de información en un sistema de oferta diaria, computarizado o telefónico, que evite los inventarios en la empresa (Ho, 1988: 127; Womack, Jones y Roos, 1990:62); y se organiza a través de un esquema de subcontratación en cascada, pues de un contrato se cuelgan subcontratos de primer, segundo y hasta tercer nivel (Lazerson, 1990; Castillo y Ramírez, 1992:38-9).

La oferta es variada porque el sistema de producción es flexible, y el sistema de producción es flexible porque la "demanda variable" es la característica de los mercados y del diseño de la estructura de producción. Así, la flexibilidad de las empresas para reducir o aumentar su producción y costos de ingeniería ante los cambios en la demanda, es lo que orienta la organización de los sistemas de producción flexible. El formato de producción es flexible porque no es único y reconoce diferentes tipos de mercado por tamaño y estacionalidad. El primer formato es de acuerdo con el tamaño del lote en unidad simple, lote pequeño, lote medio, lote grande y producción continua. Un segundo método se determina según si el proceso es continuo o intermitente. Un tercero se refiere al tipo de productos y tamaño de los lotes en pequeñas escalas de producción de una amplia variedad de productos, escal as medias de producción de un limitado rango de productos y gran escala de producción de una limitada variedad de productos. Este método indica que un amplio lote de producción permite un costo unitario del producto más bajo. Sin embargo, como la demanda y la competencia en el mercado no siempre permiten la producción en gran escala, la respuesta tecnológica más adecuada es la pequeña escala de producción de una amplia variedad de productos, conocida también como manufactura flexible. El cuarto y quinto método están relacionados con la producción por pedido, tanto en su forma de orden de trabajo como de producción para stock. La orden de trabajo implica pequeños lotes de producción, mientras el stock se refiere a grandes lotes o producción continua. Ambas formas se utilizan de manera intercambiable, dependiendo de la tendencia en el mercado (Ogawa, 1984:13-15).

Por último, el "trato al cliente" es parte de un sistema de información que permite al consumidor indicar sus decisiones al distribuidor y éste al proceso (Johanson y Nonaka, 1991:51). Siendo el distribuidor parte del sistema productivo, la información del consumidor y las posibilidades 
tecnológicas de respuesta son lo que ordena el plan de producción. Esto hace sentir a los consumidores como parte importante de la empresa (Womack, Jones y Roos, 1990:67), lejos del modelo de dominio de las preferencias del consumidor que caracteriza los esquemas orientados por la producción en masa.

\section{APRENDIZAJE INDUSTRIAL Y FUNCIÓN DE PRODUCCIÓN}

El aprendizaje en el trabajo se ha convertido en una explicación importante de las diferencias observadas en lo que se ha llamado la eficiencia X de las empresas y la economía (Mookherjee y Ray, 1991:144). Drucker ha señalado que en la sociedad actual, "el conocimiento es el principal recurso para los individuos y la economía. Tierra, trabajo y capital, los tradicionales factores de la producción, no desaparecen, pero se convierten en secundarios" (1992:95). La eficiencia que surge del aprendizaje es sustancial, como lo demuestra la experiencia japonesa de aprender a trabajar en equipo, lo cual tardaron en lograr cerca de 15 años. Sin embargo, una vez que lo hicieron, disminuyeron el tiempo de desarrollo del producto a dos tercios del tiempo estandar (Drucker, 1992:102). Como se ve, esta eficiencia X tiene que ver con la productividad que es posible lograr por encima de la productividad técnicamente posible con la función de producción elegida o típica de la empresa o la economía; y está influida por todo tipo de factores que permiten el mejor aprovechamiento de los recursos existentes. Esto es lo que da sentido a la idea de que la tecnología no sea sólo cuestión de máquinas y herramientas, y que por tanto, la innovación tecnológica se relacione con el diseño y la calidad de los materiales usados, la organización de la producción y el empaque del producto (Dijk, 1992:6).

Si la eficiencia X resulta del esfuerzo discrecional de quienes trabajan al interior de una organización, entonces el aprovechamiento óptimo de los recursos supone fallas de mercado que impiden lograr el máximo nivel de eficiencia previamente. Esta situación ilustraría el hecho de que si las empresas u otras organizaciones no mercantiles fallan. en utilizar sus recursos eficientemente, se debe a que los costos de transacción no se superan debido a la existencia de problemas de acción colectiva al interior de la empresa (Nabli y Nugent, 1989:19). Lall ha enfatizado que el desarrollo de las capacidades emprendedoras, organizacionales y tecnológicas de las empresas, depende de su capacidad de aprender internamente y de la capacidad del Estado para generar políticas orientadas a remediar las fallas del mercado que afectan su posibilidad de aprender (1990: 12,17 y 20 ). 
En el primer sentido, Garvin afirma que las empresas que mejoran es porque tienen un compromiso con el aprendizaje, mientras que las que permanecen igual tienen una ausencia del mismo. Una empresa comprometida está en capacidad de resolver problemas sistemáticamente, de experimentar con nuevas alternativas, de aprender de las propias experiencias e historias pasadas, de aprender de las experiencias y mejores prácticas de otros y de transferir, rápida y eficientemente, el conocimiento a través de la organización. En general, una empresa empieza a aprender cuando deja de preocuparse tan sólo de los costos y empieza a hacerlo por la calidad, entrega a tiempo e introducción de nuevos productos y procesos (1993:78,81 y 89). O como diría Kay, cuando aprende a resolver un mismo problema en más de una forma (1991:48).

En el segundo sentido, Lall enfatiza que las transacciones entre empresas y entre industrias, frecuentemente están marcadas por fallas de mercado. Ello se debe a que los mercados, estrechos y fragmentados, no necesariamente proveen el tipo de información que los agentes necesitan para diseñar, producir o invertir. En consecuencia, las empresas utilizarán la informacion y capacidad disponible para adquirir la maestría tecnológica y organizativa que les permita alcanzar la máxima producción. Una vez logrado este esfuerzo tecnológico, la competencia induce a las empresas a mejorar su tecnología, reducir sus costos y adaptar los productos a las necesidades del mercado. Sin embargo, cuando la maestría tecnológica y la capacidad de innovación para enfrentar mercados más competidos y riesgosos, no caracteriza ampliamente a las empresas de un país, es necesario establecer nichos de mercado internamente protegidos que absorban los costos de aprendizaje de las empresas, mientras son inducidas a entrar en los mercados externos. Lall abunda que frente a mercados de trabajo y de capital que sufren de falta de información y riesgo moral; mercados de tecnología sumamente dinámicos e imperfectos y; transacciones entre industrias con alto riesgo moral e información asimétrica, la solución para el desarrollo de las capacidades de las empresas no se encuentra en una intervención estatal al estilo de la de los modelos sustitutivos de importaciones. Más bien, en una estrategia de industrialización orientada a las exportaciones, pues con una protección adecuada, induce a un más rápido y eficiente aprendizaje tecnológico y organizativo. En otras circunstancias, en países en vías de desarrollo, sólo las grandes empresas podrían construir este tipo de capacidades tecnológicas, pues las deficiencias de mercado y la insuficiencia de redes de proveedores, les obligan a internalizar funciones que en otras condiciones podrían externalizarse. Sin embargo, en ambientes macroeconómicos donde los mercados de capital, información, calificaciones y otros mercados están 
bien desarrollados y donde las tecnologías son relativamente estables, las empresas pequeñas puede salir adelante sin sufrir en la formación de sus capacidades tecnologicas (1990:23-25). Para el caso coreano y taiwanés, es evidente que para que las grandes empresas, estatalmente apoyadas, crearan redes de subcontratación con pequeñas empresas, fue clave de su vinculación al mercado exterior (Cumings, 1988:70).

Tanto Garvin como Lall sostienen que el sistema educativo, complementado con programas de entrenamiento, tiene un significativo impacto sobre la capacidad de aprendizaje de las empresas, el aumento de su eficiencia X y el desempeño industrial agregado (Garvin, 1993:88; Lall, 1990:27). En consecuencia, el desarrollo institucional de organizaciones industriales, centros de capacitacion, intermediarios financieros, bolsas de subcontratación, etcétera, es vital para que la acción colectiva disminuya los costos de transacción e información de las empresas.

\section{PEQUEÑA EMPRESA Y ESCALA ÓPTIMA REDUCIDA}

Por décadas, las empresas en casi todo tipo de industrias han buscado economías de escala, acuciadas por la idea de que fabricando o distribuyendo bienes en grandes volúmenes, disminuyen los costos unitarios. El predominio de la producción en masa tuvo dos consecuencias en la organizacion de la producción: que los costos adicionales de expandir las empresas apenas fueran importantes y que la asociación entre gran empresa y eficiencia fuera aceptada sin discusion. La hipótesis shumpeteriana de que existe una relación positiva entre innovación y poder monopolico, y que por tanto, las grandes empresas son más innovadoras que las pequeñas, orientó las acciones de política industrial a desestimar la importancia de las pequeñas empresas en el proceso de innovación y crecimiento económico.

El ambiente recesivo de los años ochenta generó grandes sorpresas en la teoría económica. Las razones globales que ayudan a explicar el ascenso de las pequeñas empresas de los países desarrollados, son las mismas que se pensó harían más amplia la brecha entre grandes y pequeñas. En el plano del comercio, por ejemplo, la caída de las barreras comerciales ayudo a que las pequeñas pudieran actuar en mercados internacionales sin tener que enfrentar los problemas de empleados, legislación e impuestos propios de los negocios internacionales, que antes sólo podían enfrentar las grandes empresas. En el plano financiero, la amplia desregulación de los mercados de capital, permitió que las pequeñas empresas pudieran acceder al crédito en condiciones similares a las grandes antes de la desregulacion. En el plano productivo, el uso de las computadoras han venido estrechando las 
economías de escala. La automatización de fábricas ha favorecido la producción de bienes en bajos volúmenes y con costos unitarios iguales o por abajo de lo que es posible obtener con producción en gran escala. A la vez, les ha permitido utilizar las mismas técnicas logísticas, sofisticados modelos financieros, nómina automatizada, técnicas de control de calidad y otras tareas administrativas que antes sólo estaban disponibles a las grandes empresas. En el plano del consumo, la convergencia internacional de hábitos de consumo y la generalizada aplicación de técnicas de control de calidad, han afectado el poder de las grandes marcas, a saber, el más preciado activo de las grandes empresas de producción en masa (The Economist, 1993:13).

En esquemas de especialización flexible, la industria de pequeña escala ha probado su fuerza económica y tecnológica, aun en tiempos de crisis (Schmitz, 1990:280). Aunque las nuevas tecnologías han cambiado las leyes que gobiernan las economías de escala en la empresa, planta y el nivel de producto (Schmitz, 1990:281), no es previsible que "puedan conducir a drásticos cambios en la concentración de las industrias de producción en masa" (Archibugi, Cesaratto y Sirilli, 1991:311). Se deduce entonces que una estrategia de desarrollo tecnológico para las pequeñas empresas, debe tomar en cuenta no sólo la trayectoria tecnológica previa en relación con la que se desea alcanzar, tal como ha sido señalado por Dosi (1988:1130). Pratten concluye que si las pequeñas empresas pueden producir más rentablemente en relación con el capital que emplean, las firmas tenderán a ser pequeñas. Sin embargo, si las pequeñas empresas lo pueden hacer tan rentablemente como las grandes, entonces ambos tipos de empresas tenderán a coexistir (1991:31). En consecuencia, las empresas pequeñas deben considerar como parte de su estrategia de desarrollo, la coexistencia necesaria con formas diferenciales de organización industrial y fuentes de conocimiento tecnológico y organizacional. Muchas empresas grandes ya lo están haciendo, buscando reorganizarse para disminuir sus costos, la burocratización, la inflexibilidad y el desperdicio de recursos. Para ello están recurriendo, más que a nuevas compras, a nuevas formas de colaboración con otras grandes y pequeñas empresas a través de alianzas, inversiones conjuntas y subcontratación para llegar a nuevos mercados.

\section{LAS REDES DE SUBCONTRATACIÓN COMO ALTERNATIVA DE ORGANIZACIÓN INDUSTRIAL}

En términos formales, una empresa subcontratista es aquella que acepta una orden de trabajo de otra empresa, que a su vez, tiene un contrato firmado con algún cliente (Balaña, 1991:83). Según Lazerson, 
la subcontratación adquiere la forma comercial, que se caracteriza porque la empresa contratante se limita a vender y distribuir los productos terminados por la subcontratante a través de su propia red de ventas; pero también adquiere la forma industrial, que se caracteriza porque el contratante mismo está comprometido con la producción, y los componentes, partes, subensambles o manufacturas ensambladas por la subcontratante son incorporadas dentro de un producto final que él venderá como si fuera propio (1990:3).

Desde fines de los sesenta, las actividades de subcontratación han venido adquiriendo gran importancia para promover la organización-desintegración horizontal de la producción hacia las medianas y pequeñas empresas (Castillo y Ramírez, 1992:37). La descentralización de la función de producción ha reorganizado las economías de escala, pues las posibilidades tecnológicas permiten obtener en pequeños talleres, similares niveles de eficiencia que en las grandes empresas. De igual manera, la organización laboral en pequeñas empresas reduce el riesgo de conflictos y el control de calidad puede ser realizado si se controla el proceso de ensamble final o si se envían supervisores a las subcontratantes (Lazerson, 1990:9-11; Ruíz D., 1993:529).

$\mathrm{Si}$ a través de relaciones mutuamente ventajosas para las partes, la subcontratación facilita la colaboración entre grandes y pequeñas empresas; y las economías de escala no requieren de actividades en gran escala, entonces la combinación de tamaños de empresas organizadas a través de redes de subcontratación, podría ser institucionalmente organizada por una adecuada política industrial que mitigara las fallas de mercado. Al ser parte del esquema institucional de organización industrial, las relaciones entre empresas establecidas a través de la subcontratación, permiten ampliar la flexibilidad de la empresa grande, e incentivar el aprendizaje industrial de largo plazo entre las medianas y pequeñas, motivando constantes procesos de innovación. En un contexto deeconomía abierta, esta política industrial permitiría consolidar una base industrial nacional competitiva.

$\mathrm{La}$ organización industrial japonesa a través de redes de subcontratación en cascada, puso los nivelesde competitividad de su planta industrial en los más altos niveles. La subcontratación japonesa en gran escala, fue producto de una estrategia industrializadora impulsada por el gobierno. Esta se caracterizó por una alta eficiencia organizativa para construir una estructura de división del trabajo que interrelacionó a las grandes con las medianas y pequeñas empresas, a partir de un esquema de grandes empresas madres que subcontratan a cientos de medianas empresas proveedoras del primer nivel, que a su vez subcontratan a miles de pequeñas empresas proveedoras del segundo nivel, que a su vez subcontratan a miles de micro 
empresas proveedoras del tercer nivel (Lazerson, 1990:4 y 15; Castillo y Ramírez, 1992:38). Como la mayoría de las empresas pequeñas y medianas sirven como subcontratistas o proveedores a las grandes empresas, constituyen una parte integrante del formato de produccion y son sujetas a control por éstas o las compañías asociadas. Al decir de Ruíz Durán,

en su búsqueda de una combinación óptima de producción, la gran empresa mantiene grandes plantas para los procesos indivisibles que requieren de economías de escala, y subroga el resto a empresas medianas, pequeñas y micro, lo que da origen a un nuevo perfil de producción industrial más eficiente por la vía de la especialización flexible (1992:168).

Una empresa tenderá a expandir su estructura de producción, hasta que los costos de organizar una transacción extra dentro de la misma, sean iguales a los costos de realizar la misma transacción por medio de un intercambio en el mercado o a través de subcontratación. En consecuencia, así como la integración vertical en la empresa tiende a buscar ahorros de costos frente a la inflexibilidad del mercado, la desintegración vertical tiende a darse cuando los costos de las transacciones internas tienden a ser mayores que los costos de las transacciones externas realizadas a precios de mercado (Scott, 1990:35-6). Como el proceso de desintegracion vertical está en función directa de la incertidumbre y alta competitividad de los mercados, se orientará por cambios organizacionales más que tecnológicos; es decir, las redes entre empresas que disminuyan el costo global de las transacciones, serán preferibles a las jerarquías internas (Scott, 1990:53-4). Si la función de costo total medio de una empresa depende del nivel de integración o desintegración vertical y espacial, en el caso de integración total, el productor no puede enfrentar las variaciones del mercado y tendrá sobre o subcapacidad de capital y trabajo si la demanda baja o sube sobre su capacidad tecnologica de respuesta. Si la empresa se desintegra a través de la subcontratación con el fin de enfrentar la incertidumbre asociada a la apertura de la economía por ejemplo, flexibilizará su tecnología a través de un cambio organizacional que le posibilite establecer una red de subcontratistas que permitan responder a la demanda del mercado sin incurrir en costos de transaccion adicionales (Scott, 1990:55-7).

La reorganización del proceso del trabajo es un desafío que tiene que ver no sólo con los cambios tecnológicos, sino también con la reeducación laboral de quienes trabajan en el nuevo esquema de organización industrial. Tanto dentro como entre las empresas, se trata de enfrentar los choques externos y mejorar la productividad de todos, orientados por los 
beneficios que es posible obtener de la competencia por los mercados, principalmente los de exportacion (Marsh y Mannari, 1990:15-16). El principal reto para las grandes empresas, especialmente para las norteamericanas, consiste en establecer la relación entre invéstigación y desarrollo con las actividades manufactureras (Meredith, 1987:30; Jordan, 1991; Tyre, 1991:64); entre las empresas y sus subcontratistas; los trabajadores de planta y los supervisores; y los trabajadores y la tecnología (Harrison y Kelley, 1991:54).

La colaboración industrial se sustenta en una visión de los productos no como

actores autónomos, sino integrados a un proceso de alianzas estratégicas y de coinversiones, tanto a nivel nacional como internacional. Estas redes no se circunscriben a los proveedores o clientes, que resuelvan problemas financieros y de mercado y accedan a asistencia técnica y legal para mejorar sus contratos de venta y su técnica productiva (Smith, Dickson y Smith, 1991:460).

"También considera a los competidores, a las universidades y a los laboratorios de investigación del propio gobierno" (Ruíz y Kagami, 1993:6). En este sentido, la colaboración puede ser vista como una forma de integración horizontal, donde las empresas que operan en actividades similares o relacionadas, establecen acuerdos conjuntos para intercambio de tecnología e información (Smith, Dickson y Smith, 1991:458). Esta horizontalidad de la cooperación, organizada sobre todo a través de la subcontratación, introduce en el análisis de la competencia el equilibrio dinámico entre grandes y pequeñas, así como la mentalidad organizacional del beneficio (Ranfla, comunicación personal, 1993).

\section{LA REGIÓN FRENTE A LA ORGANIZACIÓN INDUSTRIAL DE REDES EMPRESARIALES}

Por la especialización propia de las economías de escala que les es posible obtener con su tamaño y su consiguiente nivel de integración vertical, las grandes empresas presionan los salarios al alza y obstaculizan el desarrollo de otras industrias. Ello obliga a un alto nivel de importaciones regionales, tanto de insumos productivos como de bienes requeridos por la poblacion. En consecuencia, hay poco comercio entre las pocas empresas de la región, pues la mayor parte del comercio es con el exterior de la misma. En suma, "la red interna de comercio y relaciones económicas decae y las relaciones de un limitado número de grandes firmas con el mundo exterior tienden a dominar la economía" 
(Steindl, 1984:9). Este tipo de desarrollo regional sin cadenas productivas y con una pequeña industria de subsistencia, ha sido ampliamente cuestionada.

El exitoso desarrollo de los distritos industriales italianos, donde la cercana localización de muchas pequeñas empresas y su organización a través de la subcontratación, les permite aprovechar ventajas de aglomeracion para responder competitivamente y con rapidez a las irrupciones de mercado. De ahí que su capacidad de especialización flexible no se asocie tan sólo al hecho de su tamaño pequeño, sino también a su capacidad para aglomerarse sectorialmente y organizarse a través de relaciones de subcontratación. Estas características técnicas y organizacionales, es lo que permite aprovechar el potencial de la eficiencia colectiva y la flexibilidad de su tamaño (Schmitz, 1990:273 y 281).

Estos distritos industriales se han caracterizado por una activa presencia de la comunidad y las empresas en un sistema productivo geográficamente definido, donde sus intereses tienden a unirse (Becattini, 1990:36). Como un gran número de empresas están involucradas entre sí en varias etapas y de diferentes formas en la producción de un producto homogéneo, complementan su producción como insumo de una red productiva conectada a diferentes tipos y segmentos de mercado a través de la subcontratación. Por ello es que una de sus características más relevantes es que una gran proporción de las empresas son pequeñas y pueden revertir su debilidad estructural, integrándose ellas mismas en una amplia red de cooperación interempresarial. Esto les permite obtener economías de escala y de alcance similares a las que por su tamaño pueden tener las grandes empresas (Dijk, 1992:6). Como el distrito industrial es un espacio productivo regional donde también las interacciones sociales y políticas que se dan en la comunidad son importantes, su éxito económico en materia de crecimiento y generación de empleo, tiene mucho que ver con los aspectos institucionales del sistema. A su vez, sugieren cómo las profundas brechas tecnológicas pueden disminuirse a través de nuevas formas de organización económica, pero también cómo las políticas estatales y las estructuras sociales pueden influenciar la forma de organización de la economía (Lazerson, 1990:16).

Al diagnosticar que una "política de largo plazo requiere la creación de un nuevo balance industrial en la region", Steindl (1984:12-13) propone que a través de la acción estatal se coordinen esfuerzos basados en un cuidadoso estudio que contemple, por un lado, las posibilidades técnicas ya existentes o en avanzado estado de desarrollo, y por otro, las capacidades disponibles en la region. Esto supone asignarle un importante peso al factor 
educativo y a la tradición empresarial reunidos localmente, para orientarlos en torno a un ambiente institucional que promueva la coordinación empresarial interna a través de la subcontratación.

La subcontratación como práctica institucionalmente organizada, ha permitido grandes éxitos industriales en Alemania, donde organizaciones sociales como la Federación de Industrias Alemanas, juegan un importante papel en el equilibrio de los intereses de sus miembros, sobre todo cuando los grandes contrastes intentan transferir a sus proveedores el precio y presión de la reorganización (Willemsen, 1993:569-570). En Corea, bajo el concepto de que las pequeñas empresas enfrentan los mismos retos y problemas de las grandes, estando menos preparadas que ellas, se ha justificado la acción estatal para su promoción. Al inicio las filiales no recibían ayuda significativa de las matrices y estaban obligados a suministrar sus productos a una sola empresa matriz. A partir de que en 1978 se revisó la Ley de promoción a la subcontratación a las pequeñas empresas, se promovieron estímulos especiales en materia de crédito, promoción frente a inversión extranjera y asistencia técnica, a empresas seleccionadas en áreas y/o productos seleccionados; se ampli6 el periodo contractual de un año a tres; y se desarrollo el sistema horizontal de subcontratación, que permite a las filiales sacar provecho de la producción en gran escala y promover la especialización, aspecto fundamental de la calidad (Baek, 1993:564; Regnier, 1993:33). En Taiwan, el gobierno alentó la formalización de redes de subcontratación por considerar que tales asociaciones de carácter semipermanente, generaría ventajas intrínsecas a las empresas integradas sin obstruir las ventajas derivadas de la independencia. Para 1991 existían 74 redes con 1,368 empresas (Chen, 1993:570).

La pregunta que realiza Chen sobre ¿por qué las empresas están dispuestas a sacrificar independencia a cambio de una mayor escala de producción?, tiene al menos dos respuestas: la primera de carácter técnico-económico, es por las economías de escala que se requieren construir en el ámbito microeconómico. La segunda tiene que ver con el ámbito macroeconómico y la necesidad de complementar los efectos adversos que sobre el capital y el trabajo han tenido las políticas depresivas de libre mercado. La subcontratación, al funcionar como una estructura organizacional que permite acceder a las grandes empresas a servicios productivos contratados interna o externamente (González-Aréchiga, Ramírez y Aguas, 1991:235), institucionalmente organizada permitiría manejar los factores de demanda y costos necesarios para prevenir los impactos depresivos y mejorar la distribución del ingreso (Kregel, 1993:16-7). 


\section{ECONOMÍA Y MAQUILADORAS EN LA FRONTERA NORTE DE MÉXICO}

El crecimiento economico de la frontera norte de México, dio lugar a importantes discusiones sobre su capacidad explicativa de los procesos de apertura que desde mediados de los ochenta se llevan a cabo en el país. Ello se debe a la fuerte influencia que en su crecimiento tuvieron sus actividades económicas con el exterior. Las zonas y perímetros libres que para su desarrollo se establecieron a fines de los treinta, fueron conformando un escenario de economía abierta que la diferenció en mucho del modelo económico de protección industrial que se adoptara para el país en su conjunto. Las ventajas fiscales y aduaneras para el comercio, la inversion industrial vía maquiladoras y el abastecimiento de bienes de consumo e insumos productivos, caracterizaron a esta parte del país y las subregiones que la conforman, por su gran dinamismo industrial basado en la subcontratación, una fuerte economía de servicios, un sector agropecuario moderno y, en general, con un gran ambiente para la generación de negocios (Mungaray, 1988; Fernández, 1989).

Este dinamismo económico dio lugar al surgimiento de amplios sectores de micros y pequeños empresarios. Un buen número de ellos iniciaron su proceso de aprendizaje industrial, tanto como producto de procesos formativos en las plantas maquiladoras de origen norteamericano, o como producto de actividades exitosas de transformación desarrolladas dentro del llamado sector informal. Se puede decir que el permanente contacto entre la tradicional creatividad mexicana y la práctica cultura de negocios norteamericana, propiciaron un fértil ambiente para el desarrollo de las actividades industriales en los estados de la frontera norte de México. La información de INEGI indica que en los estados fronterizos se concentra el $16 \%$ de las micro y pequeñas empresas y el $80 \%$ de las plantas maquiladoras del país. A su vez, capta el $80 \%$ de los empleos generados por las maquiladoras y el $83 \%$ de la derrama salarial.

En 1980, los estados del norte de México, con el $45 \%$ de la extensín territorial del país, albergaban el $16 \%$ de la población nacional y generaba el 20\% del producto interno bruto (Baker, et al. 1988:48). Para 1990 , el peso relativo de la población se mantiene, pero su partícipación en la generación del producto interno bruto se incrementó a 29\%, con una tasa anual de crecimiento de $4.2 \%$, superior a la media nacional de $2.7 \%$. Más aún, el $33 \%$ de las empresas con mayor valor exportado y el $49 \%$ de los parques y zonas industriales del país, se localizan en la región (Gaona, 1991). Una importante explicación de este desarrollo urbano e industrial se encuentra en la escasez de agua, que puso límites al 
crecimiento de la agricultura y dio lugar al desarrollo urbano e industrial (Fernández, 1989:111).

Las condiciones de apertura con el exterior que hoy orientan las acciones de política económica en México, le deben mucho a las experiencias exitosas del sudeste así́tico y a las distintas experiencias de desarrollo regional exitosas que han ocurrido en los distritos industriales; ambas con una industrialización orientada al exterior. Pensando hacia adentro, la experiencia de economía abierta de la frontera norte, es también un importante antecedente (Verkoren y Hoenderdos, 1988:35). Sin embargo, es evidente que las actividades de subcontratación internacional no profundizaron la formación empresarial de la zona debido al carácter contradictorio del modelo maquilador respecto al modelo industrializador del pais (Tamayo, 1993:69). La escasa integración de insumos nacionales a las maquiladoras de exportación, refleja problemas de información de integración, pero también la poca competitividad de la industria mexicana, organizada oligopólicamente a través de un modelo cerrado de sustitución de importaciones (González-Aréchiga y Ramírez, 1989:881). Durante muchos años, las restricciones a la competencia actuaron como restricciones a la innovación en las pequeñas empresas. Los altos costos de operación de la economía, sólo permitieron que las grandes empresas con operaciones en gran escala, pudieran enfrentar los costos y trasladarlos a precios. El sistema de protección industrial no estimuló la competencia o la colaboración entre empresas como forma de competencia. Además, dado el tamaño y concentración urbana del mercado interno, las empresas pequeñas difícilmente podrían realizar los procesos de aprendizaje que les ayudaran a mejorar en áreas críticas y desarrollar procesos de crecimiento. En estas condiciones, el modelo maquilador de economía abierta, si bien estuvo en posibilidades de contribuir a las políticas macroeconómicas de empleo y generación de divisas, difícilmente armoniza con políticas de formación empresarial y capacitación del trabajo en alto nivel. De hecho, el modelo norteamericano de maquila no se orienta a la formación de redes de pequeños proveedores nacionales para abastecer a las grandes y estables plantas maquiladoras norteamericanas.

Para que la actual estrategia de crecimiento pudiera tener los efectos positivos esperados sobre la distribución del ingreso de la región y del país, requiere de un sector empresarial más amplio y dinámico que participe con mayores niveles de innovación en la creación o asimilación creativa de tecnologías apropiadas. Sobre esta base es necesario repensar las pequenas empresas, que existen como formas de subsistencia articuladas con la cconomía popular, y sólo en casos excepcionales como entidades innovadoras vinculadas a la economía moderna (Ruíz Durán, 1992:163). 
Fomentarlas y apoyarlas tiene que ser parte de un planteamiento estratégico sobre el papel que la formación de fuerzas empresariales tendría dentro de una estrategia que busque el crecimiento con equidad (Ruíz Durán, 1991:40; CEPAL, 1990). Sin embargo, como las fuerzas empresariales no surgen en forma espontánea, una necesaria política industrial debería fomentar su surgimiento y financiar su aprendizaje en áreas geográficamente definidas y con posibilidades de ser entrelazadas en una red productiva con base en la subcontratación y/o la aglomeración a partir de las actuales plantas maquiladoras. Ello permitiría que el aprendizaje en el manejo de la tecnología a través de las actividades de maquila, no sólo sirva para aliviar la pobreza y mantenerla al nivel de subsistencia (James, 1985:118), sino para dar el salto tecnológico que les permita pasar del esquema de ventajas comparativas al de ventajas competitivas en un ambiente regional. También se requiere redefinir la amplia zona norte y sus subregiones, en redes institucionales de produccion industrial a través de una adecuada política industrial. Esto permitiría fomentar aún más la actividad empresarial, aprovechando la amplia experiencia de subcontratación que desde 1964 se ha desarrollado en la zona norte de México.

En el futuro se encuentra la necesidad de una política industrial que aproveche las actividades de subcontratación con doble objetivo: que estimule el aprendizaje empresarial en las pequeñas empresas y que apoye las estrategias de desarrollo de las más grandes. Una política de esta naturaleza favorecería el crecimiento global de la economía a través del crecimiento de la actividad empresarial y la disminución de los costos de transacción. Además, tendría gran impacto en la distribución del ingreso, pues a través de nuevas alternativas de organización flexible entre grandes y pequeñas, les permitiría a las primeras enfrentar las cambiantes condiciones del mercado, y a las segundas, acelerar su aprendizaje industrial abatiendo los costos y riesgos de tal proceso. Parece existir concenso en que una política industrial tiene sentido cuando es necesario resolver las fallas del mercado para la asignación eficiente de los recursos (Odagiri, 1986:389; Lall, 1990; Phillips, 1992:104).

Cuatro son los campos donde la política industrial tiene un gran campo de actuación: infraestructura industrial, asignación de recursos entre industrias, organización intraindustrial y pequeñas y medianas empresas (Odagiri, 1986). En todos ellos, la forma de evaluar si la política industrial es positiva o no, es a través del nivel de costos abatido con el aprendizaje empresarial o el soporte a la investigación y desarrollo; el nivel de cambio en el excedente del consumidor y el productor; y el impacto sobre otras industrias y sobre la inversión extranjera (Odagiri, 1986:394). 
Lo anterior supondría considerar a la industria maquiladora como una estructura de organización que apoya el desempeño industrial (GonzálezAréchiga, Ramírez y Aguas, 1991:235) abatiendo costos, incrementando la productividad, formando empresarios, ampliando la gama de servicios. Para ello, es evidente que se requiere una política que estimule la producción flexible, la desintegración horizontal de los procesos productivos, y la conformación de redes de subcontratación entre empresas maquiladoras grandes y pequeñas.

\section{CONCLUSIÓN}

El ascenso del sentimiento de colaboración entre gobierno y empresa que está caracterizando a las economías de fin de siglo, está pasando por la necesidad de flexibilizar la organización industrial para configurar redes productivas entre grandes y pequeñas empresas, bajo esquemas de subcontratación sujetos a mercado. Esto permite que la necesaria desintegración vertical y espacial de la empresa ante un ambiente de incertidumbre y alta competitividad, se pueda dar no ślo bajo mecanismos de mercado, sino también institucionales. El gran reto de estas redes de subcontratación, es permitir que las pequeñas empresas, sobre todo las de los países en vías de desarrollo, puedan dar el salto tecnológico que les permita generar mayor valor agregado y capitalizar (Ruíz Durán, 1993:528). Para ello, dos aspectos serán clave: que las empresas contratantes sean realmente empresas generadoras de gran valor agregado y que las empresas subcontratistas sean competitivas en términos de calidad. Esto le da a la política economica, la señal de mercado de que para impulsar las relaciones de subcontratación, es necesario impulsar el aprendizaje tecnológico en procesos, más que en productos (Ruíz Durán, 1993). Apoyar la especialización de las pequeñas empresas en procesos, les permitirá capacitarlas para que se articulen a cadenas productivas del país y del exterior (Sánchez, 1993:543). Consecuentemente y dadas las fallas de mercado originadas en la típica concentración industrial y de distribución del ingreso en las economías en vías de desarrollo, el éxito del proceso subcontratacióntransferencia-adaptación tecnológica, dependerá en gran medida de la política interna en materia de organización industrial y desarrollo tecnoIogico (Castillo y Ramírez, 1992:38).

En esencia, se trata de enfrentar las nuevas condiciones de riesgo, incertidumbre y al ta competitividad, a través de alianzas estratégicas; pero no al estilo de la subcontratación norteamericana, que Ruíz Durán ha calificado como una organización industrial basada en la competencia desleal, por su imposibilidad de ser vehículo de innovación. Esto se debe 
a que como las grandes empresas promueven la competencia entre sus proveedores para que el costo del producto se reduzca, la calidad pasa a segundo término en virtud del carácter antagónico del ambiente empresarial (1992:164). La presencia de maquiladoras japonesas y coreanas en la frontera norte de México desde mediados de los ochenta, está dando lugar a una importante red de proveedores, siguiendo el patrón con el que trabajan en sus países de origen, aunque aprovechando los bajos costos salariales, la alta productividad del trabajo, la ventaja locacional frente al mercado norteamericano y, sobre todo, aprendiendo del potencial industrial mexicano (Mungaray, 1990:155). Cuando el Tratado de Libre Comercio entre en vigor y las reglas de origen empiecen a aplicarse, este tipo de empresas habrán aprendido suficiente e integrado sus redes nacionales de proveedores. Esto les permitirá seguir compitiendo en el mercado de Estados Unidos, sólo que en mejores condiciones que las maquiladoras norteamericanas y sus socios mexicanos debido a su ventaja tecnológica y organizacional.

\section{BIBLIOGRAFÍA}

ARCHIBUGI, D., Cesaratto, S. y Sirilli, G. 1991. "Sources of innovation activities and industrial organization in Italy", Research Policy, Vol. 20 (4), agosto.

BAEK, N. 1993. "Desarrollo e incentivos para las pequeñas y medianas empresas coreanas", Comercio Exterior, Vol. 43 (6), junio.

BAILY, M.N., Burtless, G. y Litan, R.E. 1993. Growth with equity. Economic policymaking for the next century, Washington, The Brookings Institution.

BAKER, S. et al. 1988. "The magnet of growth in Mexico's north", Business Week, junio 6.

BALAÑA, A.M. 1991. "La subcontratación industrial: tendencia del comercio internacional", Comercio Internacional Banamex, Vol. 3 (1), marzo.

BARANSON, J. 1981. The japanese challenge to U.S. industry, Lexington, Lexington Books.

BECATTINI, G. 1990. "The marshallian industril district as a socio-economic notion", en F. Pyke, G. Becattini y W. Sengenberger (Eds.), Industrial district and inter-firm coopetarion in Italy, Ginebra, International Institute of Labour Studies.

CASTILLO, V.M. y Ramírez, R.J. 1992. "La subcontratación en la industria maquiladora de Asia y México", Comercio Exterior, Vol. 42 (1), enero. 
CHEN, T.J. 1993. "Las empresas pequeñas y medianas en Taiwan", Comercio Exterior", Vol. 43 (6), junio.

CEPAL. 1990. Transformación productiva con equidad, Santiago.

COASE, R. 1937. "The nature of the firm", Económica, 4, noviembre.

CUMINGS, B. 1988. "The origins and development of the northeast asian political economy: industrial sectors, product cycles, and political consequences", en F. Deyo (Ed.), The political economy of the new asian industrialization, Ithaca y London, Cornell University Press.

DEUTSCHMANN, C. 1987. "Economic restructuring and company unionism: the japanese model", Economic and industrial democracy, Vol. $8(4)$, noviembre.

DIJK, M.P. Van. 1992. The interrelation between industrial districts and technological capabilities development, documento presentado en: UNCTAD/GTZ symposium of the role of industrial districts in the aplication, adaptation and diffusion of technology, Ginebra, noviembre.

DOSI, G. 1988. "Sources, procedures and microeconomic effects of innovation", Journal of Economic Literature, Vol. XXVI (3), septiembre.

DRUCKER, P. 1992, "The new society of organizations", Harvard Business Review, Vol. 70 (5), sep.-oct.

FERNÁNDEZ, R. 1989. The Mexican-american border region. Issues and trends, Notre Dame, University of Notre Dame Press.

GARVIN, D.A. 1993. "Building a learning organization", Harvard Business Review, Vol. 71 (4), julio-agosto.

GERLACH, M.L. y Lincoln, J. R. 1992. "The organization of business networks in the United States and Japan", en N. Nohria y R.C. Eccles (Ed.), Networks and organizations. Structure, form, and action, Boston, Harvard Business School Press.

GONZÁLEZ-ARÉCHIGA, B. y Ramírez, J.C. 1989. "Perspectivas estructurales de la industria maquiladora", Comercio Exterior, Vol. 39 (10), octubre.

GONZÁLEZ-ARÉCHIGA, B., Ramírez, J.C. y Aguas, F. 1991. "Maquiladoras e intercambio de servicios", en M. Gibbs y D. Díaz (Coord.), México: una economía de servicios, Reporte del proyecto $M E X / 87 / 026$, Nueva York, Naciones Unidas.

HAYES, R. y Jaikumar, R. 1988. "Manufacturin's crisis: new technologies, obsolete organizations", Harvard Business Review, Vol. 66 (5), sep-oct.

HARRISON, B. y Kelley, M.R. 1991. "The new industrial culture journey toward collaboration", The American prospect, invierno. 
HO, S.K.M. 1988. Information technology development for small and medium enterprises in Asian NICs and Japan, Tokyo, Asian Productivity Organization.

JAMES, J. 1985. "The role of appropiate technology in a redistributive development strategy", en J. James y S. Watanabe (Eds.), Technology, institutions and government, McMillan Press.

JOHANSON, J.K. y Nonaka, I. 1991. "Market research; the japanese way", en Harvard Business Review, Marketing research. The right way, Cambridge.

JORDAN, A.G. 1991. "The future of the factory", Managment Digest.

KAY, A.C. 1991. "Computer, networks and education", Scientific American, Vol. 256 (3), septiembre.

KREGEL, J.A. 1993. "Keynesian stabilization policy and post war economic performance", en A.Szirmai, B. van Ark y D. Pilat (Eds.), Explaining economic growth, North Holland, Elsevier.

LALL, S. 1990. Building industrial competitiveness in developing countries, Paris, OECD.

LAZERSON, M. 1990. Subcontracting as an alternative organizational form to vertically-integrated production, International Institute for Labour Studies, NIOP/DP/20, Ginebra.

MARSH, R.M. y Mannari, H. 1990. Organizational change in japanese factories, London, Jai Press Inc.

MEREDITH, J.R. 1987. "The strategic advantages of the factory of the future", California Managment Review, Vol. XXIX (3), primavera.

MOOKHERJEE, D. y Ray, D. 1991. "El aprendizaje en el trabajo y la estructura industrial: un panorama", El Trimestre Económico, Vol. LVIII (1), enero-marzo.

MUNGARAY, A. y Álvarez, J. 1987. "La oferta de espacios industriales en el desarrollo de Tijuana", FONEP, núm. 123, enero.

MUNGARAY, A. 1988. "Internacionalidad del desarrollo regional de la frontera norte de México, 1960-1985", Foro Internacional, Vol. XXIX (1), julio-septiembre.

MUNGARAY, A. 1990. Crisis, automatización y maquiladoras, Mexicali, Universidad Autónoma de Baja California.

MUNGARAY A. 1991. "Maquiladoras y desarrollo industrial en la frontera norte de México", en R. Pozas y M. Luna (Coords.), Las empresas y los empresarios en el México contemporáneo, México, Grijalbo.

NABLI, M.K. y Nugent, J.B. 1989. "The new industrial economic development: an introduction and concluding remarks", en M.K. Nabli y J.B. Nugent (Eds.), The new institutional economics and 
development. Theory and applications to Tunisia, Amsterdam, North Holland.

NONAKA, I. 1991. "The knowledge company", Harvard Business Review, Vol. 69 (6), nov.-dic.

OGAWA, E. 1984. Moden production managment, a Japanese experien$c e$, Tokyo, Asian Productivity Organization.

OBRINSKY, M. 1983. Profit theory \& capitalism, Philadephia, University of Pennsylvania Press.

ODAGIRI, H. 1986. "Industrial policy in theory and reality", en H.W. de Jong y W.G. Shepherd (Eds.), Mainstreams in industrial organization-Book II, Dordrecht, Kluwer Academic Publishers.

PHILLIPS, K.P. 1992. "U.S. Industrial policy: inevitable and ineffective", Harvard Business Review, Vol. 70 (4), julio-agosto.

PRATTEN, C. 1991. The competitiviness of small firms, Cambridge, Cambridge University Press.

REGNIER, P. 1993. "The dynamics of small and medium-sized enterprises in Korea and other Asian NIEs". Small Business Economics, 5 .

ROTHWELL, R. 1987. "Las pequeñas y medianas empresas, motor de la innovación", Economía Informa, núm. 156, sep.

RUIZ Durán, C. 1991. "Desarrollo y globalización: en busca de un nuevo paradigma", Economía Informa, núm. 200, nov-dic.

RUIZ Durán, C. 1992. "Las empresas micro, pequeñas y medianas: crecimiento con innovación tecnologica", Comercio Exterior, Vol. 42 (2), feb.

RUIZ Durán, C. 1993. "México: crecimiento e innovación en las micro y pequeñas empresas", Comercio Exterior, Vol. 43 (6), junio.

RUIZ Durán, C. y Kagami, M. 1993. Potencial tecnológico de la micro y pequeña empresa en México, México, Nacional Financiera.

RUIZ Veraza, H. 1991. "Transferencia de tecnología en la industria maquiladora electrónica de Tijuana", El Economista Mexicano, Vol. XXI (1).

SÁNCHEZ Ugarte, F. 1993. "Acciones en favor de las micro, pequeñas y medianas industrias en México", Comercio Exterior, Vol. 43 (6), junio.

SCHMITZ, H. 1990. "Small firms and flexible specialisation in developing countries", Labour and Society, Vol.15 (3).

SCOTT, A.J. 1990. Metropolis. From the division of labor to urban form, Berkeley, University of California Press.

SMITH, H.L., Dickson, K. y Smith, S.L. 1991. "There are two sides to every story: innovation and collaboration within networks of large and small firms", Research Policy, Vol. 20 (5), octubre. 
STEINDL, J. 1984. "Structural problems in the present crisis", Trieste, mimeo, septiembre.

STORPER, M. 1985. "Oligopoly and the product cycle: essentialism in economic geography", Economic Geography, Vol. 61 (3).

TAMAYO, J. 1993. "The maquila industry in perspective", Journal of Borderland Studies, Vol. VIII (1).

TYRE, M.J. 1991. "Managing innovation on the factory floor", Technology Review, Vol. 94 (7), octubre.

VERKOREN, O. y Hoenderdos, W. 1988. "La política industrial en México y la industrialización en la zona fronteriza del norte de México", Estudios Fronterizos, núm. 15-16, ene-ago.

WILLEMSEN, A. 1993. "Pequeñas y medianas empresas: la experiencia alemana", Comercio Exterior, Vol. 43 (6), junio.

WOMACK, J.P., Jones, D.T. y Roos, D. 1990. The machine that changed the world. The story of lean production, New York, Harper-Perennial.

\section{Periódicos}

GAONA, J.L. 1991. "Seis estados fronterizos serán el eje de la economía mexicana", El Economista.

The Economist. 1993. "The fall of big business", abril 17. 